\title{
Algorithms for the selection of fluorescent reporters
}

\author{
Prashant Vaidyanathan ${ }^{1,+}$, Evan Appleton ${ }^{4,5,+}$, David Tran ${ }^{6, \mathrm{x}}$, Alexander Vahid ${ }^{7, \mathrm{x}}$, George \\ Church $^{4,5}$, and Douglas Densmore ${ }^{2,3, *}$
}

\author{
${ }^{1}$ Biological Computation group, Microsoft Research, Cambridge, CB1 2FB, UK \\ ${ }^{2}$ Department of Electrical and Computer Engineering, Boston University, Boston, 02215, USA \\ ${ }^{3}$ Biological Design Center, Boston University, Boston, 02215, USA \\ ${ }^{4}$ Harvard Medical School, Department of Genetics, Harvard University, Boston, 02115, USA \\ ${ }^{5}$ Wyss Institute for Biologically Inspired Design, Harvard University, Boston, 02115, USA \\ ${ }^{6}$ Alexa Al - Natural Understanding, Amazon, Cambridge, 02142, USA \\ ${ }^{7}$ Amazon Global Logistics Tech, Amazon, Boston, 02210, USA \\ *dougd@bu.edu \\ +These authors contributed equally to this work \\ xThese authors contributed equally to this work
}

\begin{abstract}
Molecular biologists rely on the use of fluorescent probes to take measurements of their model systems. These fluorophores fall into various classes (e.g. fluorescent dyes, fluorescent proteins, etc.), but they all share some general properties (such as excitation and emission spectra, brightness) and require similar equipment for data acquisition. Selecting an ideal set of fluorophores for a particular measurement technology or vice versa is a multidimensional problem that is difficult to solve with ad hoc methods due to the enormous solution space of possible fluorophore panels. Choosing sub-optimal fluorophore panels can result in unreliable or erroneous measurements of biochemical properties in model systems. Here, we describe a set of algorithms, implemented in an open-source software tool, for solving these problems efficiently to arrive at fluorophore panels optimized for maximal signal and minimal bleed-through.
\end{abstract}

\section{Introduction}

Fluorescence-based measurement of biological systems was first introduced in the 1960s and has since grown to be used as a method to measure biochemical properties in virtually every model organism ${ }^{1}$. These measurements have allowed researchers to probe the expression levels of proteins and other molecules at distinct points in time and space. Fields such as immunology, neuroscience, and synthetic biology make heavy use of these probes, as it is often critical to track expression of multiple signals within a sample. As such, fluorescence-based measurement has become the cornerstone of qualitative measurement technologies ${ }^{2,3}$, quantitative measurement technologies ${ }^{4}$ and modern sequencing technologies ${ }^{5,6}$. These technologies are built by composing expensive, precise mechanical equipment that relies upon lasers and detectors to measure fluorescence.

For all of these modern technologies, one key challenge is to maximize the number of different signals (i.e. colors) that can be distinguished in a single measurement in order to maximize the number of independent probes that can be used simultaneously, which can subsequently minimize the number of experiments and associated costs. However, choosing independent probes for a specific application or experiment is not trivial because many fluorescent probes emit light spectra that overlap with one another, making it difficult to separate signals from different probes (this problem is referred to as 'spectral spillover', 'bleed-through', or 'crossover'). To solve for potential bleed-through, makers of measurement machines often build in more lasers and detectors. While this gives the biologist more flexibility to choose probes, the onus is still on the user to resolve the trade-offs like bleed-through between certain probes. Fortunately, this problem of bleed-through can be corrected for by applying a series of linear algebraic operations by a process known as spectral compensation ${ }^{7}$. While the process of correcting spectral spillover using compensation is generally considered solved and easy to implement in theory, there are many nuances that make it difficult to implement in practice ${ }^{8}$. If compensation is misunderstood or misapplied, it can lead to incorrect biological measurements. As biologists perform experiments that need to be able to accurately resolve many fluorescent probes simultaneously ${ }^{9-13}$ (where often greater than 10 orthogonal probes are required), choosing the right set of fluorophores and corresponding detectors is crucial while designing experiments to ensure correct analysis of the resulting data ${ }^{12}$.

In general, all fluorescence measurement technologies rely on the excitation of biochemical compounds with a laser (or other light source) at a certain wavelength and the capture of light emitted from these compounds across a spectrum of wavelengths using mirrors, filters, and light detectors. Current methods of designing large high-dimension flow cytometry panels involve 
painstaking ad hoc methods that involve computing spillover coefficients ${ }^{14,15}$, analyzing gating strategies, and cycling through the latest literature to make qualitative-based selections. This is compounded by the fact that there isn't a 'one shoe fits all' solution for selecting $n$ fluorophores out of a universal set of available fluorophores. This is primarily due to the fact that every measurement machine has a unique configuration (i.e. set of lasers and associated detectors). Exploring every possible panel design might be intractable depending upon the size of the panel (as shown in Fig 2A). Hence, biologists and laboratories might be limited to panel designs that have already been studied and published or might have to limit the solution space based on an intuitive understanding of the emission and excitation spectra of the fluorophores available, to design a panel. The unfortunate consequence is that newly discovered fluorophores may not be easily incorporated in experiments since it may cause unintended spillover, forcing biologists to redesign panels from scratch.

To address this challenge, we created a computational solution housed in an open source software tool to solve the following problem - given a measurement instrument where the laser and detector configurations are known, and a library of fluorophores under consideration, design an optimized $n$-color panel of fluorophores by maximizing the amount of signal in each detector and minimizing the amount of bleed-through. We also present a way to compare the optimality of two panels and present search algorithms to explore the solution space, find valid panel designs, and identify the best possible n-color fluorescence panel for a given instrument and collection of fluorophores. We demonstrate that our heuristic algorithm can reliably search for an optimal fluorescence panel even from a very large solution space. We also demonstrate that our computational predictions of signal and bleed-through reliably match experimental observations.

\section{Results \\ FPselection}

To solve the flourophore selection problem, we have designed an open-source web-application and command-line tool, to allow users to design an n-color panel for a specific measurement instrument - http://fpselection.org/. Our software uses search algorithms to explore the solution space of all possible n-panel configurations that can be constructed from a library of fluorophores for a fluorescence measurement instrument to find optimal panel configurations. The tool allows users to upload a file containing a list of fluorophores with excitation and emission spectra, an instrument configuration file, and choose a value for $n$ to find valid and optimal n-color panels. Users can optionally upload files containing the brightness of each flourophore (Section S2.1.3), or include the autofluorescence of the cell line used for the expression of the fluorescent proteins. Additional information regarding the format of these files can be found in Supplemental Section S2.1.

Since there are no established benchmarks for optimality of cytometry panels, we chose the following two properties to optimize an n-color panel: first, the amount of signal measured by a detector, for the fluorophore it is supposed to detect; and second, the amount of bleed-through from all other fluorophores in that detector. A panel configuration is considered as 'valid' if each detector in the panel measures a non-zero amount of signal for the fluorophore it is intended to measure. (see S1.1 for details on how predictions of signals and bleed-through are calculated for each detector in a panel). Finding a valid panel configuration is non-trivial as highlighted in Fig. 1, where the probability of randomly selecting a valid panel of 4 fluorophores out of a library of 8 for an instrument with 16 detectors is only $0.7 \%$. An additional complexity is that not all valid panels can measure fluorescence efficiently. For example, Fig. 1 shows a valid panel containing a detector which has very low signal and high bleed-through from other fluorophores. One possible solution would be to use a larger library of fluorophores to get better valid panels that are optimal. However, the solution space increases exponentially with the increase in the size of the fluorophore library. For instance, the number of possible solutions to design a 4-color panel for the same measurement instrument used in Fig. 1 using all the available fluorophores listed in FPBase ${ }^{16}$ (which has 734 fluorophores listed) is $5.23 \times 10^{14}$, which is clearly intractable. However, an algorithmic approach can find a reliable fluorophore panel from a reasonably large solution space. To solve for this problem, FPselection uses the search algorithms to find a valid panel where the amount of signal in each detector is maximized and bleed-through is minimized.

Fig. 2A shows that as the size of $n$ increases, and as the number of detectors in the cytometer increases, the percentage of valid panel configurations also significantly reduces. For instance the probability of randomly choosing a valid 2-color panel out of a library of 12 fluorophores for an instrument with 7 detectors is $21.75 \%$. However, the probability of randomly choosing a valid 2-color panel from the same library for an instrument with 19 detectors is $10.17 \%$ and the probability of choosing a valid 6-color panel at random for the same instrument is $0.04 \%$. 


\section{Algorithm Overview}

\section{Searching for optimal n-color fluorophore panels}

We designed various search algorithms to explore the search space of n-color cytometry panels for a given set of fluorophores and instrument configurations. Supplemental section S1.4 contains the psuedo-code and performance metrics of these algorithms. Our recommended algorithm uses simulated annealing to quickly and reliably find an optimal result. The tool runs 50 concurrent threads, where each thread spawns a simulated annealing run, and the best result among the 50 threads is returned as the solution. This is particularly useful when the solution space is huge which can be due to larger fluorophore libraries, larger number of detectors in the measurement instrument, or larger values of $n$.

We tested the effiencity of our search algorithm by comparing it against an exhaustive list of valid panels for 3 different flow cytometers (BD LSRFortessa, Miltenyi MACSquant VYB, and CytoFlex LX) with varying and unique sets of lasers and detectors with the same sample sources to get experimental replicates. The detectors in all these cytometers were photomultiplier tubes (PMTs) with varying bandpass filters (Section S4). For each cytometer, we performed 200 runs of simulated annealing to find $\mathrm{n}$-color panels out of a library of 8 fluorophores (Section S3), with the panel size ranging from 2 to 5 . We then compared these results to an exhaustive list of all valid fluorophore panels ranked from best to worst, based on the criteria specified in the Methods section. The panel size and size of the library was restricted due to computational memory limitations which was encountered while sorting the exhaustive list of valid panel designs.

We observed that the run-time for simulated annealing was constant and each run took less than 1 second to complete (as opposed to exhaustive search where the run-time increases exponentially with panel size and the size of the library and set of available detectors, and can take hours or days based on the size of the solution space - Fig.S2). We also observed that simulated annealing typically returned the optimal solution in most runs. For instance, Fig.2B shows the trajectories of 200 runs of simulated annealing to search for 5-color panels, from a library of 8 fluorophores for a measurement instrument with 19 detectors. This test had the largest solution space, where there were 78,140,160 5-color panels of which 449,762 were valid. Simulated annealing was able to find a valid solution in all 200 runs and was able to find the best solution 191 times. The average rank of the solutions found by simulated annealing was 2.005 with a standard deviation of 15.718 . This indicates that the simulated annealing approach performs reliably well as a heuristic and generally produces an optimal result.

\section{Comparing experimental observation and computational predictions}

To validate our computational predictions, we performed an experiment over-expressing our library of 12 fluorescent proteins in human induced pluripotent stem cells (hiPSCs). We determined the signal and bleed-through values in each detector of the top 100 optimal n-color panels for all three cytometers for values of $n$ ranging from 2 to 5 . The signal and bleed through (experimental) measurements and (computational) predictions in each detector were normalized to 1 based on the highest measured/predicted value respectively. In an ideal panel, the normalized value of the measured or predicted signal in each detector should be 1 while the normalized bleed-through values from other fluorophores should be as close to 0 as possible.

To compare the computational predictions against experimental measurements, we considered 2 basic metrics: 1 . the number of panels where, for all detectors in the panel, the fluorophores with normalized values equaling 1 matched; and 2. the number of panels where for all detectors in the panel, the difference between each normalized prediction and measurement value must be within $0.05(5 \%), 0.10(10 \%)$, and $0.20(20 \%)$ of one another. For instance, in Fig.3D the signals match for all detectors, since for each detector, the fluorophore signal measurement and prediction values are 1 . Hence, we say that the signals match for this panel. Similarly, the difference between normalized values of the signals and bleed-through are within 0.05 of each other (and hence by extension within 0.10 and 0.20 of each other) except the bleed-through from mPlum in the Yel/Green Laser's Detector C, where the normalized measurement and predictions have a difference of 0.123 . Hence we say that this panel is within 0.20 but not within 0.05 or 0.10 .

We started with a library of 12 fluorophores (see Sectoion S3 for the list of fluorophores used), where the excitation and emission spectra and brightness was obtained from FPbase and other sources ${ }^{16-19}$. The term brightness, refers to the molecular brightness of a fluorophore which is the product of the molar extinction coefficient and the quantum yield of the fluorophore ${ }^{16}$. We normalized the brightness of the fluorophores to the brightest fluorophore - tdTomato (S1.1). When we compared the predictions and measurements, we noticed that while predictions generally matched for 2 and 3 color panels, there was a significant drop for 4 and 5 color panels. We also noticed that the predictions matched more closely for the LSRFortessa and the CytoFlex (which had more lasers and detectors) as compared to the MACSquant which only had 3 lasers and 7 detectors.

Since the molecular brightness may not reflect the actual brightness observed in the experiments ${ }^{16}$, we decided not to scale the emission of the fluorophores based on brightness for the next run. The predictions without brightness were significantly better than the previous run, especially for larger panel sizes. This is highlighted in Fig.3A, 3B, and 3C. 


\section{Discussion}

This work presents a set of algorithms that solve a key experimental design problem routinely re-solved by bench scientists - the selection of fluorescent reporters based upon the measurement device available in the lab. We have demonstrated that for small libraries of fluorophores on simple devices, we can do a reliable exhaustive search for an ideal set of fluorophores (Fig. S4), and that for a large library of fluorophores on a complex device, we can use fast heuristics that still perform very well to select an optimal set of fluorophores (Fig.2B) for an n-color panel. Furthermore, to verify that our algorithms were working properly and selecting reasonable solutions, we validated that fluorescent proteins expressed in human iPSCs had highly-matching results for overlap matrices compared to the algorithm-predicted outcomes (Fig. 3D). The fluorophores used in this case study were fluorescent proteins. However, this work can very easily be applied to any type fluorophore (such as fluorescent dyes and conjugated antibodies) as long as the emission and excitation spectra are available.

While we did not go into depth in how additional optimizations of fluorophore selection can be made for other physical properties such as oligomerization, protein maturation, etc., the ability to weight these considerations exists in the algorithmic framework. Another way to do this in the current tools without any sophisticated optimizations already is simply to consider smaller subsets of fluorophores that are relevant to the particular experiments being performed. Future work could also be done with more sophisticated parameters of specific machines that have non-linear behavior in terms of laser strength, PMTs sensitivity, etc., but this work might have diminishing returns and become highly specific to each machine. However, since the software tool is open source, the code can easily be modified to tailor the results to account for specific biochemical behaviors and instrument parameters.

In summary, we demonstrated that we can make fast, highly-optimized, and accurate decisions about which fluorophores to use for a given experiment on a given machine. This tool will serve a need that has existed in the bio-science community for a long time - countless bench scientists have had to solve this exact problem over and over again with varying degrees of proper background knowledge, possibly leading to many unfortunate selections of fluorophores for experiments that hurt their reliability and reproducibility. While the validation in this study was done using flow cytometers for their quantitative readouts, these algorithms are just as relevant for any microscope or sequencing technology that uses lasers to read out fluorescent signal. We think that this new resource will save countless hours of work re-solving this problem for a multitude of experiments and yield better choices of fluorophores for experiments.

\section{Methods}

\section{Comparing two n-color panels}

We developed a method to compare two n-color panels, to assess the optimality of multi-color fluorescence panels. The panels are compared using the following 3 properties in the order specified below:

1. Number of detectors where bleed-through is within some threshold $(\eta)$. We define a threshold $\eta$ as an acceptable percentage of bleed-through in a detector from other fluorophores that can be tolerated compared to the signal of the fluorophore it is supposed to detect. A detector is said to have bleed-through within $\eta$ if, the sum of all the bleed-through in that detector is within $\eta \%$ of the signal measured by that detector. The panel with the most number of detectors where bleed-through is within $\eta$ is considered better. If this value is the same for both panels, we use the next property. For our case studies, we chose the value of $\eta$ as $10 \%$ (the value of $\eta$ can be modified in the code).

2. Geometric mean of signals - We use geometric mean since this describes the "central tendency" of a values in a data set better. By using geometric mean over arithmetic mean, we avoid choosing cytometry panels where a single signal value measured may be large but the other values are too small. The panel with the larger geometric mean of signals is considered better. If this value is the same for both the panels, we move to the last property.

3. Arithmetic mean of bleed-through observed in the filters. The total bleed-through in a detector is computed by adding the bleed-through from all other fluorophores of the panel. The mean bleed-through is computed by calculating the arithmetic mean of the total bleed-through in all the detectors of the a panel. The panel with the smaller arithmetic mean of bleed-through is considered better. If both panels have the same value, we consider both panels equivalent.

\section{Cell line creation}

Human iPS cell lines were used for expression of fluorescent proteins. Fluorescent protein DNA sequences were cloned into a plasmid backbone that had an upstream doxycycline (DOX)-inducible pTRET promoter and a downstream puromycin expression cassette. Genomic integration sites flanked these components on the plasmid. These plasmids were nucleofected into iPS cells and then cells containing the fluorescent proteins cassettes were selected with puromycin (Sigma Aldrich). These 
cell lines were expanded in mTeSR (StemCell Technologies) and then induced with DOX for four continuous days. Cells were then digested with TrypLE (Gibco) and fixed in BD Cytofix buffer (BD Biosciences), washed in DPBS (Gibco), and stored at $4 \mathrm{C}$ for up to one week.

\section{Flow cytometry}

Fixed iPS cell lines were re-suspended in DPBS (Gibco) and run through 3 flow cytometers: (1) BD LSRFortessa [5 lasers — 16 channels]; (2) Miltenyi MACSquant VYB [3 lasers - 7 channels]; and (3) CytoFlex LX [5 lasers - 19 filter channels]. At least 10,000 events per sample were acquired within the dynamic range of the PMTs. Spectral overlap was calculated using the R Bioconductor package.

\section{Acknowledgements}

The authors would like to thank Luis Ortiz for his extensive feedback for the manuscript. The authors would also like to acknowledge Radhakrishna Sanka, Marilene Pavan, Calin Belta, and Nicholas DeLateur for their valuable feedback and insight. This work was supported by NSF grant \#1522074 and \#1446607 and the DARPA ELM Program under contract W911NF-17-2-0079.

\section{Contributions}

E.A., P.V., and D.D. conceived the project. P.V., D.T, A.V., E.A, and D.D. designed the software. E.A. designed and performed experiments. P.V. and E.A analyzed the results. P.V., E.A., D.T., and A.V, were students of Boston University and part of the Biological Design Center at Boston University when they started working on this project. All authors wrote and reviewed the manuscript.

\section{References}

1. Shaner, N. C., Steinbach, P. A. \& Tsien, R. Y. A guide to choosing fluorescent proteins. Nature methods 2, 905 (2005).

2. Hell, S. W. \& Wichmann, J. Breaking the diffraction resolution limit by stimulated emission: stimulated-emission-depletion fluorescence microscopy. Optics letters 19, 780-782 (1994).

3. Rajadhyaksha, M., Grossman, M., Esterowitz, D., Webb, R. H. \& Anderson, R. R. In vivo confocal scanning laser microscopy of human skin: melanin provides strong contrast. Journal of Investigative Dermatology 104, 946-952 (1995).

4. Fulwyler, M. J. Electronic separation of biological cells by volume. Science 150, 910-911 (1965).

5. Canard, B. \& Sarfati, R. S. Dna polymerase fluorescent substrates with reversible 3-tags. Gene 148, 1-6 (1994).

6. Lee, J. H. et al. Highly multiplexed subcellular rna sequencing in situ. Science 343, 1360-1363 (2014).

7. Roederer, M. Spectral compensation for flow cytometry: visualization artifacts, limitations, and caveats. Cytometry: The Journal of the International Society for Analytical Cytology 45, 194-205 (2001).

8. Roederer, M. Compensation in flow cytometry. Current protocols in cytometry 22, 1-14 (2002).

9. Mahnke, Y. D. \& Roederer, M. Optimizing a multicolor immunophenotyping assay. Clinics in laboratory medicine 27, 469-485 (2007).

10. Baumgarth, N. \& Roederer, M. A practical approach to multicolor flow cytometry for immunophenotyping. Journal of immunological methods 243, 77-97 (2000).

11. Perfetto, S. P., Chattopadhyay, P. K. \& Roederer, M. Seventeen-colour flow cytometry: unravelling the immune system. Nature Reviews Immunology 4, 648 (2004).

12. Ashhurst, T. M., Smith, A. L. \& King, N. J. C. High-dimensional fluorescence cytometry. Current Protocols in Immunology 119, 5.8.1-5.8.38 (2017).

13. Cai, D., Cohen, K. B., Luo, T., Lichtman, J. W. \& Sanes, J. R. Improved tools for the brainbow toolbox. Nature methods 10, 540 (2013).

14. Gentleman, R., Carey, V., Huber, W., Irizarry, R. \& Dudoit, S. Bioinformatics and computational biology solutions using $R$ and Bioconductor (Springer Science \& Business Media, 2006).

15. Beal, J. et al. Model-driven engineering of gene expression from rna replicons. ACS synthetic biology 4, 48-56 (2015).

16. Lambert, T. J. Fpbase: A community-editable fluorescent protein database. Nature methods 16, 277 (2019). 
bioRxiv preprint doi: https://doi.org/10.1101/2020.05.15.098186; this version posted May 16, 2020. The copyright holder for this preprint (which was not certified by peer review) is the author/funder, who has granted bioRxiv a license to display the preprint in perpetuity. It is made available under aCC-BY 4.0 International license.

17. Filonov, G. S. et al. Bright and stable near-infrared fluorescent protein for in vivo imaging. Nature biotechnology 29, 757 (2011).

18. Shaner, N. C. et al. Improving the photostability of bright monomeric orange and red fluorescent proteins. Nature methods 5, 545 (2008).

19. Bindels, D. S. et al. mscarlet: a bright monomeric red fluorescent protein for cellular imaging. Nature methods 14, 53 (2017). 


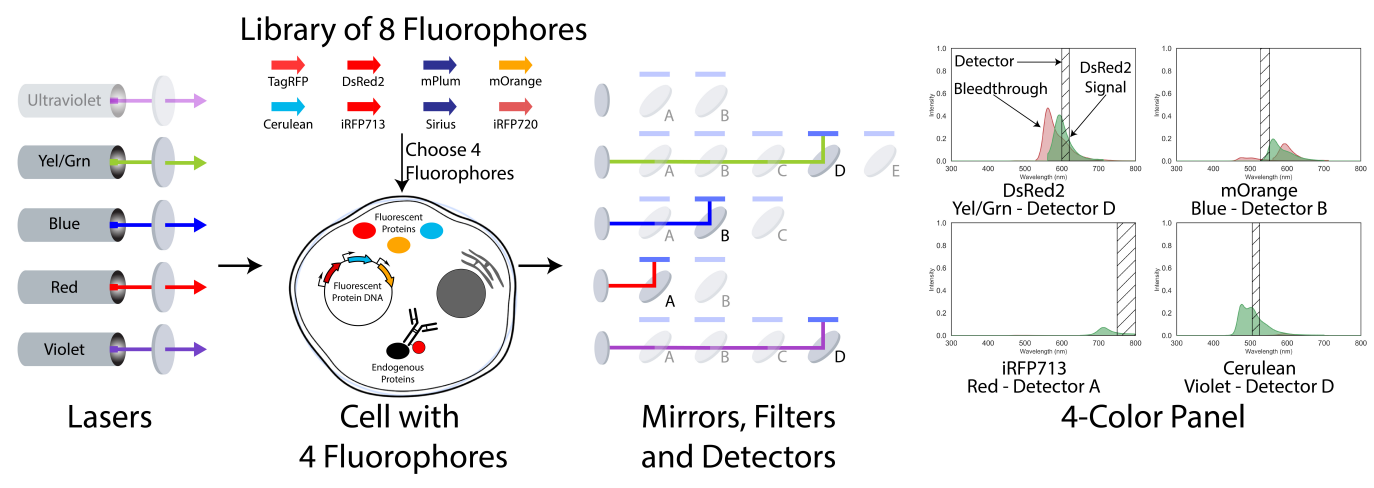

Choosing 4 out of 8 Fluorophores for an instrument with 16 detectors $=\mathbf{3 , 0 5 7 , 6 0 0 ~ 4 - C o l o r ~ p a n e l s ~}$

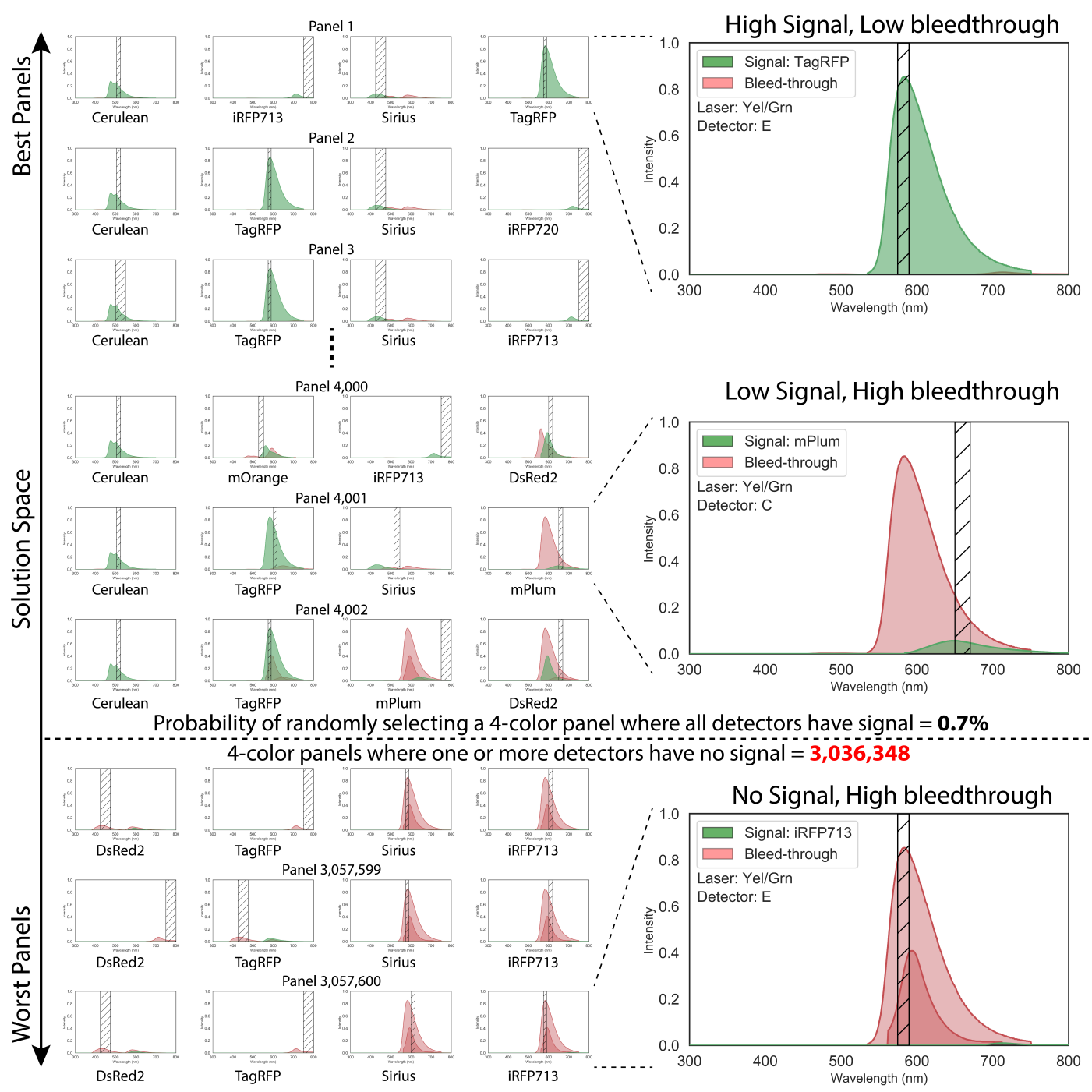

Figure 1. Selecting 4 fluorophores from a library of 8 fluorophores on a fluorometry machine with 16 detectors. (TOP) Fluorophores can include fluorescent proteins, dyes, and conjugated antibodies. The light created by illuminating these fluorophores is generally activated with lasers of specific wavelength and captured by detectors that accept certain ranges of light signal on either a microscope or PMTs. The libraries of possible fluorophores to use and possible machine settings to detect the light are vast and present a problem difficult or impossible to properly optimize using ad hoc techniques. (BOTTOM) In this case, selecting just 4 fluorophores from a library of 8 for this specific instrument is intractible since there are over 3 million possible 4-color panels, of which only $0.7 \%$ are valid. Even among the valid panels, a few configurations may be sub-optimal due to high bleed-through and low signal in the detector as shown in the middle plot on the right. Our algorithms can optimze the panel design by maximizing signal in each detector and minimizing bleed-through (like the plot on the top right). 
A

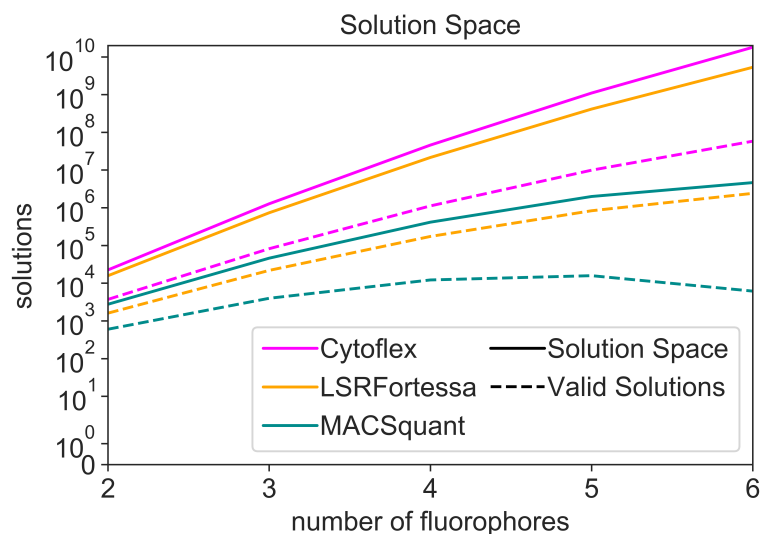

B

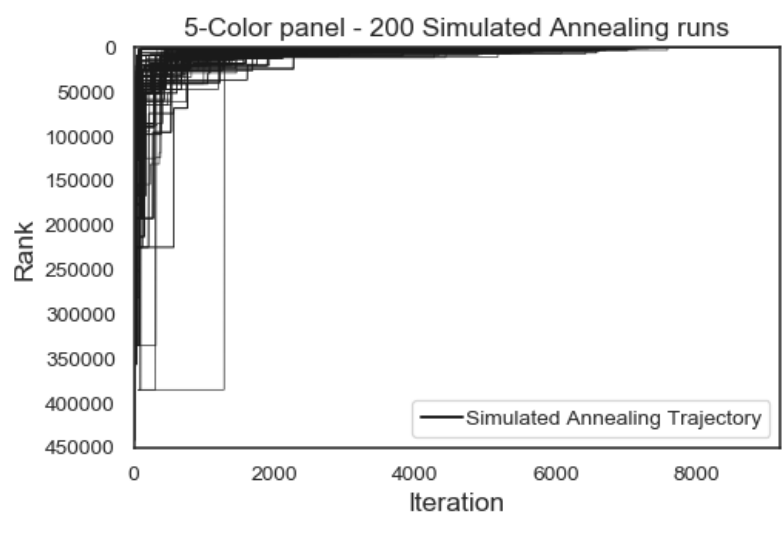

Figure 2. Computational performance of fluorophore selection algorithms. A. The number of possible solutions and valid solutions for selecting fluorophores from the library of 12 fluorophores (used in the case study) on any given machine increases exponentially with the size of the panel. This shows that for larger panel designs, it is not feasible to exhaustively search the entire solution space, and an efficient heuristic is required to find an optimal panel. B. Trajectories of 200 runs of simulated annealing to search for a 5-color panel from a library of 8 fluorophores, for an instrument with 19 detectors. Each simulated annealing run has over 9000 iterations and the plot shows the rank of the best panel at any point of time during the run. The converge to the optimal solution (the best rank) towards the end of the run. This shows that the simulated annealing approach is a reliable heuristic to find an optimal panel design. 
A

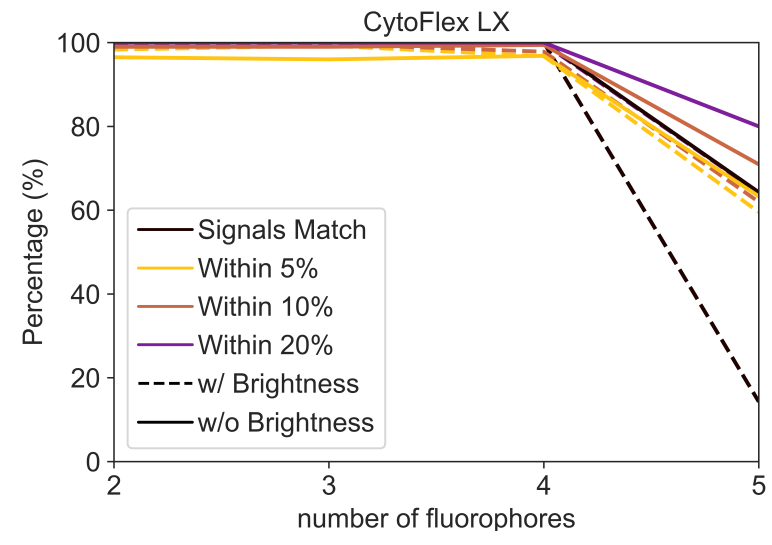

C

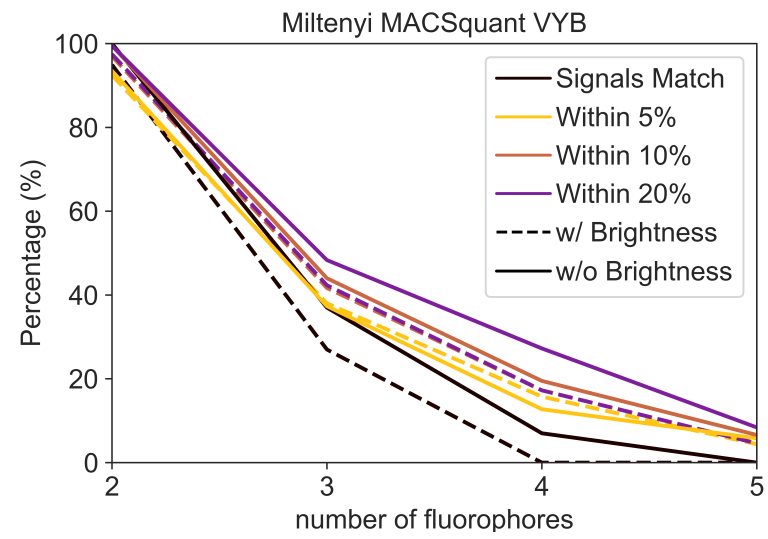

B

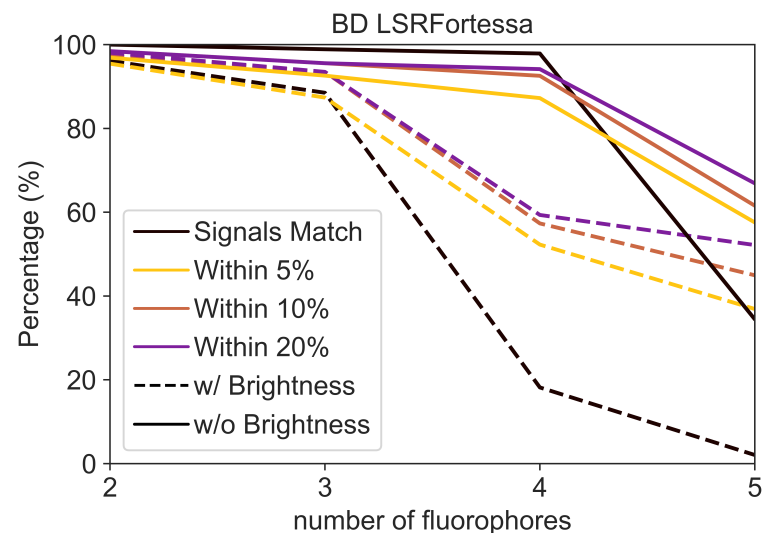

D

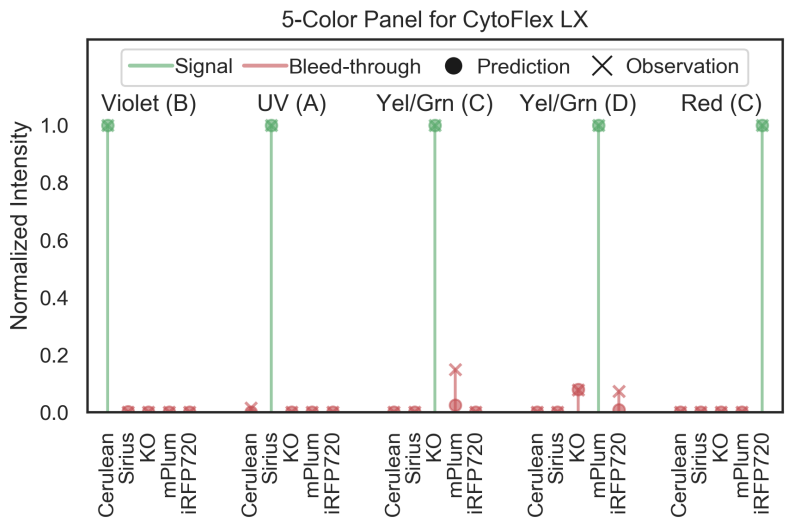

Figure 3. Experimental validation of exhaustive search fluorophore selection algorithm from a library of 12 fluorescent proteins expressed in human iPSCs on three flow cytometers. A.,B., and C., show the agreement of predicted signal/noise on a CytoFlex LX system with 5 lasers and 19 PMTs, on a BD LSRFortessa system with 5 lasers and 16 PMTs, and on a Miltenyi MACSquant system with 3 lasers and 7 PMTs respectively. The plots show the percentage of panels where the computational prediction of signal match the experimental measurements and the percentage of panels where the predicted signal and bleed-through values were within 5,10, and $20 \%$ of the experimental measurements. The predictions are better when the emission of the fluorophores are not scaled based on their brightness. These plots also indicate that for larger panel sizes, having a larger variety of lasers and filters that can excite and measure wider range of wavelengths results in better agreement with computational predictions. D. An example of the comparison between the computational predictions and experimental measurements for the best 5-color panel selected for the CytoFlex LX system, designed by fpSelection. The green lines indicate normalized signal values while the red lines indicate bleed-through from other fluorophores. In this panel, for each detector, the normalized values of signals and bleed-through match and are within 5\% of one another except for the bleed-through from mPlum in detector $\mathrm{C}$ of the Yel/Grn laser where the difference between normalized computational and experimental intensity values is $12.3 \%$. 